\title{
LncRNAs and EGFRvIll sequestered in TEPs enable blood-based NSCLC diagnosis
}

This article was published in the following Dove Press journal:

Cancer Management and Research

\section{Chang-Liang Luo',* \\ Zhi-Gao Xu',* \\ Hao Chen ${ }^{2}$ \\ Jia Ji' \\ Yu-Hui Wang' \\ Wei $\mathrm{Hu}^{\prime}$ \\ Kun Wang ${ }^{3}$ \\ Wu-Wen Zhang' \\ Chun-Hui Yuan ${ }^{4}$ \\ Fu-Bing Wang' \\ 'Department of Laboratory Medicine, Zhongnan Hospital of Wuhan University, Wuhan, China; \\ ${ }^{2}$ Department of Pathology, Zhongnan \\ Hospital of Wuhan University, \\ Wuhan, China; ${ }^{3}$ Department of \\ Laboratory Medicine, Hubei Cancer \\ Hospital, Wuhan, China; ${ }^{4}$ Department \\ of Laboratory Medicine, Wuhan \\ Children's Hospital, Huazhong \\ University of Science and Technology, \\ Wuhan, China}

*These authors contributed equally to this work

Correspondence: Fu-Bing Wang

Department of Laboratory Medicine,

Zhongnan Hospital of Wuhan University,

No 169 Donghu Road, Wuchang District,

Wuhan 43007I, China

Tel +86 27 678I 35I7

Fax +86 2767813128

Email wfb20042002@sina.com

Chun-Hui Yuan

Department of Laboratory Medicine,

Wuhan Children's Hospital, Huazhong

University of Science and Technology,

Wuhan 430016, China

Email Chunhuii.yuen@whu.edu.cn
Background: Tissue biopsy-based cancer diagnosis has limitations because of the fact that tumor tissues are in constant evolution and extremely heterogeneous. The current study was aimed to examine whether tumor-educated blood platelets (TEPs) might be a potential all-in-one source for blood-based cancer diagnostics to overcome the limitations of conventional cancer biopsy. Methods: In the present study, we evaluated the expression pattern of MAGI2 antisense RNA 3 (MAGI2-AS3) and ZNFX1 antisense RNA 1 (ZFAS1) in both plasma and platelets of 101 non-small-cell lung cancer (NSCLC) patients. Receiver operating characteristic (ROC) curve was generated to evaluate their diagnostic potential. In addition, epidermal growth factor receptor $(E G F R)$ mutations were detected in DNA and RNA samples of platelets for companion diagnostics.

Results: Our results showed that the levels of MAGI2-AS3 and ZFAS1 in both plasma and platelets of NSCLC patients were significantly downregulated than those in healthy controls. A positive correlation of long noncoding RNA expression was observed between platelets and plasma ( $r=0.738$ for $M A G I 2-A S 3, r=0.751$ for $Z F A S 1$, respectively). By ROC analysis, we found that molecular interrogation of MAGI2-AS3 and ZFAS1 in TEPs and plasma can offer valuable diagnostic performance for NSCLC patients (area under the ROC curve $[\mathrm{AUC}]_{M A G I 2-A S 3}=$ $0.853 / 0.892$, and $\mathrm{AUC}_{\text {ZFASI }}=0.780 / 0.744$ for diagnosing adenocarcinoma and squamous cell carcinoma cases from controls, respectively). Clinicopathologic characteristic analysis further revealed that $M A G I 2-A S 3$ level significantly correlated with tumor-node-metastasis (TNM) stage ( $p=0.001$ in TEPs, $p=0.003$ in plasma), lymph-node metastasis $(p=0.016$ in TEPs, $p=0.023$ in plasma), and distant metastasis ( $p=0.045$ in TEPs, $p=0.045$ in plasma), while ZFAS1 level was only correlated with TNM stage ( $p=0.005$ in TEPs, $p=0.044$ in plasma). Furthermore, EGFRvIII RNA existed in both TEPs and plasma, but EGFR intracellular mutations cannot be detected in DNA of TEPs isolated from NSCLC.

Conclusion: Our data suggested that TEP is a promising source for NSCLC diagnosis and companion diagnostics.

Keywords: tumor-educated platelets, IncRNA-MAGI2-AS3, IncRNA-ZFAS1, EGFR mutations, NSCLC

\section{Background}

Non-small-cell lung cancer (NSCLC), mainly divided into adenocarcinoma (AD) and squamous cell carcinoma (SCC) pathologically, ${ }^{1,2}$ is the predominant form of lung cancer and accounts for the majority of cancer deaths worldwide. ${ }^{3}$ Despite much progress being achieved in early detection and treatment, the 5-year survival rate for NSCLC patients is still only $5 \%-20 \% .{ }^{4}$ Thus, it is an urgent medical need to develop 
more sensitive diagnostic methods and novel biomarkers, especially for patients with recurrent cancers. ${ }^{5-7}$

Due to tumor evolution and heterogeneity, traditional tissue biopsy-based cancer diagnostic procedures have limitations in their assessment for cancer diagnosis, genotyping, and prognosis. ${ }^{8}$ Therefore, clinicians and scientists have tried to use blood-based liquid biopsies as a potential alternative diagnostic method for NSCLC patients., ${ }^{9} 10$

Liquid biopsy, including tumor-educated blood platelets (TEPs), exosomes, circulating cell-free tumor DNA, and circulating tumor cells, could offer earlier and more convenient diagnosis for patients with NSCLC. Additionally, these techniques are less invasive and may provide a more comprehensive characterization for NSCLC. ${ }^{11-13}$ The plasma is a pool of cells or cell products (DNA, RNA, and proteins) derived from the tumor or circulating tumor cells and stromal cells of the tumor microenvironment. The analysis of these blood components can, therefore, provide a comprehensive real-time information of the tumor-associated changes in an individual cancer patient. ${ }^{14}$ However, it has been also reported that tumor-associated biomarkers released into the plasma are susceptible to degradation, while blood platelets are more stable and can offer high sensitivity for the detection of EML4-ALK rearrangements. ${ }^{15}$ Given the fact that blood platelets, the second most abundant cell type in peripheral blood, can take up tumor-derived microvesicles loaded with tumor-specific cellular compounds (RNA, protein, etc), ${ }^{16}$ TEPs have been proposed and currently investigated as a potential biomarker source for cancers. ${ }^{17}$

Long noncoding RNA (lncRNA) is a group of RNA molecules that are longer than 200 nucleotides and do not encode protein, ${ }^{18}$ and they regulate gene expression through epigenetic regulation, splicing, imprinting, transcriptional regulation, and subcellular transport ${ }^{19}$ and play important roles in tumorigenesis and tumor progression. ${ }^{20}$ In recent years, aberrant lncRNA expression has been found to participate in NSCLC development and metastasis. For example, increased $H N F 1 A-A S 1$ promoted lung AD cell proliferation and metastasis through interacting with $D N M T 1$ and repressing E-cadherin expression. ${ }^{21}$ LncRNA metastasis-associated lung adenocarcinoma transcript 1 (MALAT1) is a highly conserved nuclear IncRNA, which is upregulated and can serve as a predictive marker for metastasis in lung cancer. ${ }^{22}$ These data indicated that lncRNAs play important roles in NSCLC pathogenesis, which could provide new insights into the biology of this devastating disease.

The epidermal growth factor receptor $(E G F R)$, a prototype member of the type I receptor tyrosine kinase (TK) family, plays a critical role in NSCLC cell differentiation, proliferation, and treatment response. Genetic alterations of the EGFR, including intracellular mutations such as 19-Del, L858R, T790M, 20-lns, G719X, S768I, and L861Q and extracellular mutation EGFRvIII, predominantly found in $\mathrm{ADs},{ }^{23}$ are representative biomarkers in determining the appropriate treatment for advanced lung cancer. The identification of $E G F R$ mutations provides guidance for clinical treatment with $E G F R$ TK inhibitors such as gefitinib and erlotinib. ${ }^{24,25}$

In the present study, we first screened out five lncRNAs with potential diagnostic significance from three gene expression omnibus (GEO) datasets, GSE19188, GSE30219, and GSE27262, ${ }^{26}$ which included expression information of tumor tissue from patients with AD or SCC. Next, we investigated the expression pattern and diagnostic value of five lncRNAs in platelets and plasma derived from patients with NSCLC to explore whether TEPs could enable NSCLC diagnostic and identify NSCLC types. Furthermore, we evaluated EGFR intracellular mutations in DNA extracted from platelets and plasma of 14 NSCLC patients who have been confirmed with $E G F R$ intracellular mutations in tissue biopsy samples, and we also detected EGFRvIII expression within TEPs RNA for companion diagnostic of NSCLC.

\section{Methods}

\section{Platelets and plasma isolation}

Whole-blood samples $(2 \mathrm{~mL})$ of NSCLC patients and healthy controls were collected from Zhongnan Hospital of Wuhan University under approval of Institutional Review Board, and written informed consent was obtained from all participants. Then, platelets and plasma were isolated from the same sample of whole blood by standard centrifugation within 24 hours to minimize detrimental effects of long-term storage at room temperature and decrease of platelet/plasma RNA quality and quantity. Three hundred microliters of platelets-rich plasma was obtained by centrifugation at room temperature for $20 \mathrm{~min}$ at $120 \mathrm{~g}$ to remove interference of cells. Then, platelets were isolated from the $300 \mu \mathrm{L}$ platelet-rich plasma by centrifugation at room temperature for $20 \mathrm{~min}$ at $360 \mathrm{~g}$, and resuspended in $300 \mu \mathrm{L}$ PBS after washing twice. Plasma and platelets were frozen in parallel and stored at $-80^{\circ} \mathrm{C}$ for further use.

\section{GEO lung cancer gene expression data}

Three panels of NSCLC in NCBI GEO were used in our lncRNA screening with the accession IDs GSE19188, GSE30219, and GSE27262. Reading and processing of data were done using R package "limm", genes with adjusted 
$p$-value $<0.05$ and fold change $>2$ were identified as threshold value to judge differentially expressed lncRNAs, then we proceeded to receiver operating characteristic (ROC) analysis to screen out four lncRNAs that possessed great diagnostic value (we also added an lncRNA that possessed poor diagnostic value).

\section{RNA isolation and cDNA synthesis}

Total RNA was isolated from $300 \mu \mathrm{L}$ platelet suspension and $300 \mu \mathrm{L}$ plasma using a Liquid Total RNA Isolation Kit (RP4002, BioTeke, Beijing, China) and eluted in $35 \mu \mathrm{L}$ of prewarmed $\left(65^{\circ} \mathrm{C}\right)$ elution according to the manufacturer's instruction. The concentration of RNA was measured by Nanodrop 2000 spectrophotometer (Thermo Scientific Inc, USA). Then RNA was reverse transcribed to cDNA using PrimeScript ${ }^{\mathrm{TM}}$ RT reagent kit with gDNA Eraser (RR047A, Takara, Dalian, China). Reverse transcription conditions were as follows: $42^{\circ} \mathrm{C}$ for $2 \mathrm{~min}$, and then $37^{\circ} \mathrm{C}$ for $15 \mathrm{~min}$, $85^{\circ} \mathrm{C}$ for $5 \mathrm{~s}$. The cDNA was frozen at $-20^{\circ} \mathrm{C}$ for further use.

\section{Quantitative real-time PCR (RT-qPCR) for IncRNAs}

The expression of lncRNAs was determined on the BioRad CFX96 (Bio-Rad Laboratories Inc, Hercules, CA, USA) using SYBR-Green I Premix Ex Taq following the manufacturer's instructions. The GAPDH was used as the endogenous control and was amplified simultaneously with target genes. The synthesized RT-qPCR primers are listed in Table S1. The reactions were performed in a volume of $20 \mu \mathrm{L}$ ( $10 \mu \mathrm{L}$ of SYBR mix, $0.8 \mu \mathrm{L}$ of $10 \mu \mathrm{mol}$ sense, $0.8 \mu \mathrm{L}$ of 10 $\mu \mathrm{mol}$ antisense, $2 \mu \mathrm{L}$ cDNA and $6.4 \mu \mathrm{L}$ water). The reactions started at $95^{\circ} \mathrm{C}$ for $5 \mathrm{~min}$, followed by 42 cycles of $95^{\circ} \mathrm{C}$ for $30 \mathrm{~s}, 63.3^{\circ} \mathrm{C}$ for $30 \mathrm{~s}$, and $72^{\circ} \mathrm{C}$ for $30 \mathrm{~s}$. All experiments were carried out in duplicate for each data point. The relative gene expression level was calculated using the comparative $\mathrm{Ct}$ method formula $2^{-\Delta \mathrm{Ct}}$.

\section{DNA extraction and amplification- refractory mutation system (ARMS)-PCR for EGFR intracellular mutations}

DNA was isolated from 14 TEPs and plasma samples using QIAamp ${ }^{\circledR}$ Circulating Nucleic Acid kits (QIAGEN, Hilden, Germany) eluted in $50 \mu \mathrm{L}$ of elution according to the manufacturer's instruction; EGFR intracellular mutations including 19-Del, L858R, T790M, 20-lns, G719X, S768I, and L861Q were detected using an ADx-ARMS EGFR mutation detection kit (ADx-EG01, AmoyDx, Xiamen, China) by ARMS.

\section{RT-PCR for EGFRvIII}

Samples from 110 AD, 95 SCC, and 50 healthy controls were collected and determined EGFR/EGFRvIII existence with RT-PCR in a volume of $25 \mu$ Lsolution $(12.5 \mu \mathrm{L}$ of AmpliTaq Gold $^{\circledR} 360$ Master mix, $0.5 \mu \mathrm{L} 10 \mu \mathrm{mol}$ sense, $0.5 \mu \mathrm{L} 10$ $\mu \mathrm{mol}$ antisense, $2 \mu \mathrm{L}$ cDNA, and $9.5 \mu \mathrm{L}$ water). PCR condition is listed as follows: $95^{\circ} \mathrm{C} 5 \mathrm{~min} ; 95^{\circ} \mathrm{C} 30 \mathrm{~s}, 55^{\circ} \mathrm{C} 45 \mathrm{~s}$, $72^{\circ} \mathrm{C} 1 \mathrm{~min} \times 42$ cycles; $72^{\circ} \mathrm{C} 7 \mathrm{~min}$. The RT-PCR primers are listed in Table $\mathrm{S} 1$.

\section{Statistical analysis}

Statistical analyses were performed using SPSS version 22.0 (SPSS, Chicago, IL, USA) or Prism6 (GraphPad software, La Jolla, CA, USA). Data were presented as median with interquartile range. The Shapiro-Wilk test was carried out to check the normality of the distribution. The normally distributed numeric variables were evaluated by Student's $t$-test, while non-normally distributed variables were analyzed by Mann-Whitney test. Chi-square test was used to analyze the categorical variables. One-way analysis of variance (ANOVA) or nonparametric test was used to validate the different expression levels of lncRNAs among subgroups. Statistical differences were set at $* p<0.05, * * p<0.01, * * * p<0.001$, and $* * * * p<0.0001 . p<0.05$ was considered statistically significant. To estimate the diagnostic value of the biomarkers, area under the ROC curve analysis was performed.

\section{Results \\ Identification of potential IncRNAs as biomarkers for NSCLC diagnosis from GEO dataset}

To identify relevant datasets, we searched GEO for NSCLC expression profiling studies, and the criteria for choice of datasets are that 1) they were gene profiling studies in patients with NSCLC; 2) they used the same platform; 3) they used NSCLC tissue and normal lung tissue for comparison; and 4) they contained the information of $\mathrm{AD}$ or SCC. We finally narrowed down and analyzed three GEO public datasets: GSE19188 containing 45 AD, 27 SCC, and 65 normal lung tissue controls, GSE30219 containing 85 AD, 61 SCC, and 14 normal lung tissue controls, GSE27262 containing $25 \mathrm{AD}$ and 25 normal lung tissue controls. Based on these three GEO datasets, we screened out five lncRNAs including MAGI2-AS3, LOC100507632, FXF1-AS1, LOC100499467, and ZFAS1 listed in Table 1. MAGI2-AS3, $L O C 100507632$, and FOXF1-AS1 were significantly downregulated $(p<0.0001$ in all three GEO datasets) in NSCLC 
Table I LncRNAs are screened out from three GEO datasets

\begin{tabular}{|c|c|c|c|c|c|c|c|c|c|c|c|}
\hline \multirow[t]{2}{*}{ LncRNA } & \multirow{2}{*}{$\begin{array}{l}\text { GEO } \\
\text { dataset }\end{array}$} & \multicolumn{5}{|c|}{ Adenocarcinoma } & \multicolumn{5}{|c|}{ Squamous cell carcinoma } \\
\hline & & AUC & $95 \% \mathrm{Cl}$ & p-value & Se (\%) & Sp (\%) & AUC & $95 \% \mathrm{Cl}$ & p-value & Se (\%) & Sp (\%) \\
\hline \multirow[t]{3}{*}{ MAGI2-AS3 } & GSEI9I88 & 0.921 & $0.863-0.980$ & $<0.0001$ & 82.2 & 98.5 & 0.985 & $0.967-1.003$ & $<0.0001$ & 100 & 87.7 \\
\hline & GSE30219 & 0.945 & $0.895-0.994$ & $<0.0001$ & 88.2 & 92.7 & 0.986 & $0.963-1.008$ & $<0.0001$ & 98.4 & 92.9 \\
\hline & GSE27262 & 0.972 & $0.947-1.012$ & $<0.0001$ & 100 & 92 & - & - & - & - & - \\
\hline \multirow[t]{3}{*}{ LOCI00507632 } & GSEI9I88 & 0.950 & $0.895-1.005$ & $<0.0001$ & 100 & 84.4 & 0.992 & $0.979-1.004$ & $<0.0001$ & 90.8 & 100 \\
\hline & GSE30219 & 0.968 & $0.926-1.010$ & $<0.0001$ & 92.9 & 91.8 & 0.993 & $0.980-1.006$ & $<0.0001$ & 100 & 93.4 \\
\hline & GSE27262 & 1.000 & $1.000-1.000$ & $<0.0001$ & 100 & 100 & - & - & - & - & - \\
\hline \multirow[t]{3}{*}{ FOXFI-ASI } & GSEI9I88 & 0.928 & $0.864-0.992$ & $<0.0001$ & 93.9 & 91.1 & 0.989 & $0.972-1.005$ & $<0.0001$ & 93.9 & 100 \\
\hline & GSE30219 & 0.945 & $0.876-1.015$ & $<0.0001$ & 85.7 & 96.5 & 0.972 & $0.936-1.008$ & $<0.0001$ & 85.7 & 98.4 \\
\hline & GSE27262 & 1.000 & $1.000-1.000$ & $<0.0001$ & 100 & 100 & - & - & - & - & - \\
\hline \multirow[t]{3}{*}{ LOCI00499467 } & GSEI9I88 & 0.887 & $0.809-0.9645$ & $<0.0001$ & 96.9 & 82.2 & 0.995 & $0.987-1.003$ & $<0.0001$ & 96.9 & 100 \\
\hline & GSE30219 & 0.857 & $0.740-0.9745$ & $<0.0001$ & 71.4 & 91.7 & 0.968 & $0.927-1.010$ & $<0.0001$ & 85.7 & 96.7 \\
\hline & GSE27262 & 0.992 & $0.977-1.007$ & $<0.0001$ & 96 & 96 & & & - & & \\
\hline \multirow[t]{3}{*}{ ZFASI } & GSEI9I88 & 0.665 & $0.560-0.771$ & 0.0033 & 64.4 & 64.6 & 0.660 & $0.527-0.793$ & 0.016 & 63 & 69.2 \\
\hline & GSE30219 & 0.668 & $0.540-0.797$ & 0.0436 & 61.2 & 78.6 & 0.609 & $0.479-0.739$ & 0.0497 & 57.4 & 78.6 \\
\hline & GSE27262 & 0.781 & $0.644-0.9174$ & 0.0007 & 92 & 68 & - & - & - & - & - \\
\hline
\end{tabular}

Abbreviations: AUC, area under the ROC curve; GEO, gene expression omnibus; Se, sensitivity; Sp, specificity.

tissue, while LOC100499467 ( $p<0.0001$ in all three GEO datasets) and ZFAS1 ( $p<0.05$ in all three GEO datasets) were significantly upregulated. In addition, the first four IncRNAs all exhibited great diagnostic value (area under the ROC curve [AUC] $>0.900$ ), while the diagnostic value for $Z F A S 1$ was relatively poor.

\section{MAGI2-AS3 and ZFASI were both downregulated in NSCLC platelets and plasma}

To confirm the finding of bioinformatics analysis, we used 68 ADs and 33 SCCs and 60 healthy controls for detecting these five IncRNAs to test whether TEPs could serve as a liquid biopsy for tumor diagnosis. Our result indicted that we can only detect MAGI2-AS3 and ZFAS1 expression among five lncRNAs from TEP samples. Although LOC100507632, FOXF1-AS1, and LOC100499467 can be detected in A549 NSCLC cell line, we cannot detect their expression because of the low abundance in $300 \mu \mathrm{L}$ platelet suspension and 300 $\mu \mathrm{L}$ plasma (Figure S1). For MAGI2-AS3, both platelets and plasma were significantly downregulated in line with the result from GEO datasets (Figure 1A, B). The melt curve of $M A G I 2-A S 3$ showed that the amplification specificity was good (Figure S2A, B). The result of agarose gel electrophoresis revealed that the amplification product was the target gene $M A G I 2-A S 3$ and endogenous gene GAPDH (Figure S2C). In addition, platelets' $M A G I 2-A S 3$ expression was positively correlated with plasma MAGI2-AS3. Spearman's correlation analysis showed that the correlation coefficient was 0.738 $(p<0.0001$; Figure 1C). ZFAS1 was significantly downregu- lated in platelets and plasma samples from NSCLC, which was different from the result from GEO datasets (Figure 1D, E), and spearman correlation analysis showed that the correlation coefficient was $0.751(p<0.0001$; Figure 1F). The melt curve (Figure S2D, E) and result of agarose gel electrophoresis (Figure $\mathrm{S} 2 \mathrm{~F}$ ) both showed that the amplification specificity was good. Based on the results of MAGI2-AS3 and $Z F A S 1$, we proposed that TEPs could also detect tumor biomarkers the same as that using plasma to a certain extent.

\section{Correlation between the expression level of MAGI2-AS3 and ZFASI and clinical parameters of NSCLC in platelets and plasma}

According to the median value of relative lncRNA expression in NSCLC platelets and plasma, 101 NSCLC patients were correspondently classified into two groups; in platelets (plasma), relative high group: expression ratio $\geq$ median, and relative low group: expression ratio < median. As presented in Table 2, all clinicopathologic data were divided into these two groups based on different grouping criteria and were analyzed by chi-squared test. The clinicopathologic relevance analysis of NSCLC patients demonstrated that the $M A G I 2-A S 3$ expression significantly correlated with tumornode-metastasis (TNM) stage ( $p=0.001$ in platelets, $p=0.003$ in plasma), lymph-node metastasis ( $p=0.016$ in platelets, $p=0.023$ in plasma), and distant metastasis ( $p=0.045$ in platelets, $p=0.045$ in plasma), while ZFAS1 level was only correlated with TNM stage ( $p=0.005$ in platelets, $p=0.044$ in plasma). 

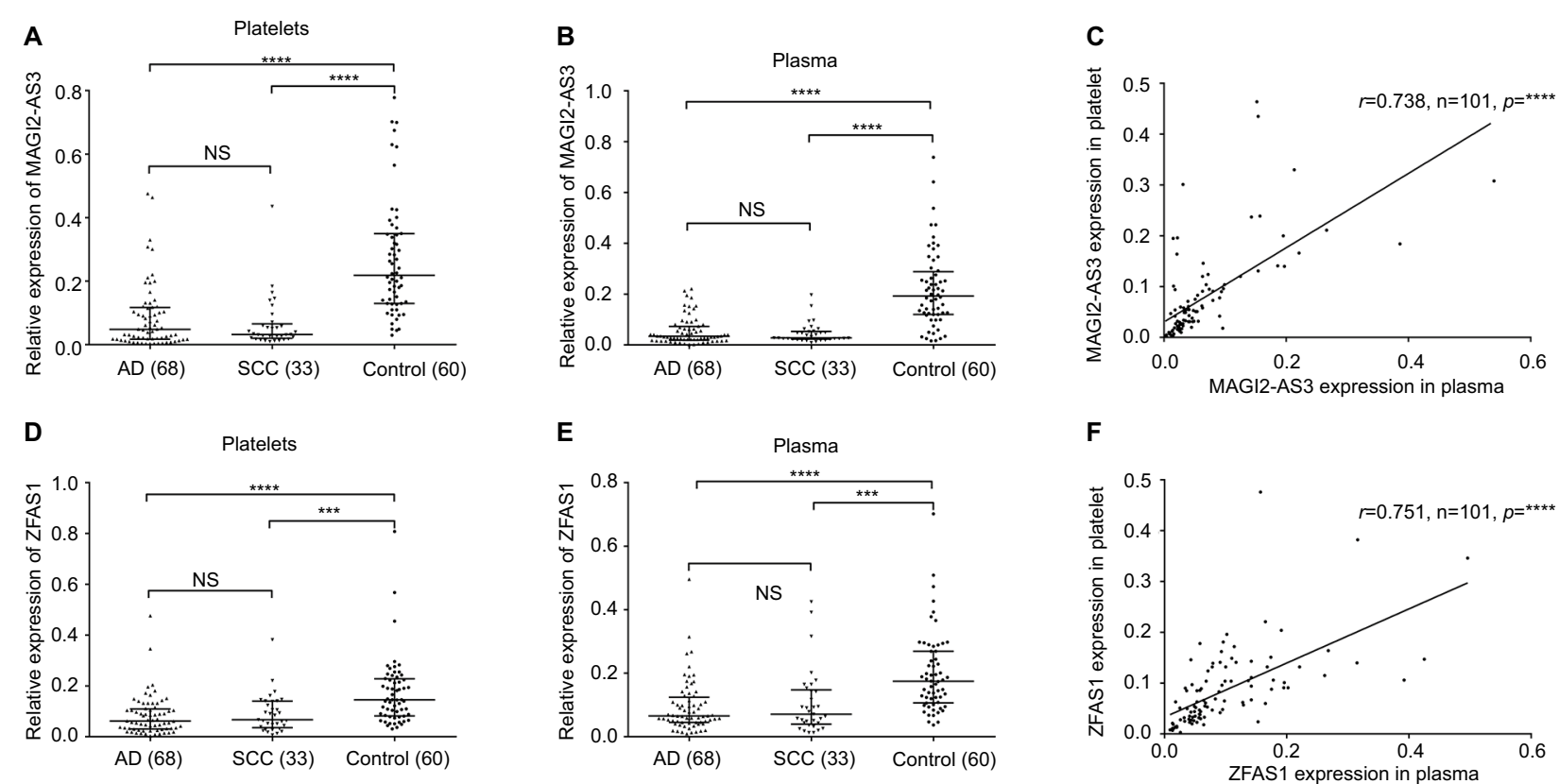

Figure I MAGI2-AS2 and ZFASI levels were downregulated in platelets and plasma samples derived from NSCLC patients.

Notes: (A) MAGI2-AS3 was downregulated in adenocarcinoma and SCC platelets than that in control platelets ( $p<0.00 \mathrm{I})$. (B) MAGI2-AS3 was downregulated in adenocarcinoma and SCC plasma than that in control plasma $(p<0.001)$. (C) Platelets' MAGI2-AS3 level was positively correlated to the plasma MAGI2-AS3 expression. The correlation coefficient was 0.738 ( $p<0.000 \mathrm{I})$. (D) ZFASI was downregulated in adenocarcinoma and SCC platelets than that in control platelets ( $p<0.00 \mathrm{I})$. (E) ZFASI was downregulated in adenocarcinoma and SCC plasma than that in control plasma $(p<0.00 \mathrm{I})$. (F) Platelets' ZFASI level was positively correlated to the plasma ZFASI expression. The correlation coefficient was $0.75 \mathrm{I}(p<0.000 \mathrm{I})$. The relative expression level was calculated using $2^{-\triangle C T}$ method; error bars represent the median with interquartile range. All data were analyzed using nonparametric test; NS, no significance. $* * * p<0.00 \mathrm{I}$, $* * * * p<0.000 \mathrm{I}$.

Abbreviations: AD, adenocarcinoma; MAGI2-AS3, MAGI2 antisense RNA 3; SCC, squamous cell carcinoma; ZFASI, ZNFXI antisense RNA I.

\section{Diagnostic value of MAGI2-AS3 and ZFASI analysis in AD and SCC}

ROC curve analysis was used to evaluate the predicting diagnosis value of lncRNAs for NSCLC. ROC was designed to use two models: AD vs controls, $\mathrm{SCC}$ vs controls. AUCs of the platelets and plasma MAGI2-AS3 were $0.853(95 \%$ $\mathrm{CI}=0.789-0.918, p<0.0001)$ and $0.866(95 \% \mathrm{CI}=0.802-0.929$, $p<0.0001)$ in $\mathrm{AD}$ (Figure $2 \mathrm{~A}), 0.892(95 \% \mathrm{CI}=0.819-0.965$, $p<0.0001)$, and $0.887(95 \% \mathrm{CI}=0.813-0.961, p<0.0001)$ in SCC (Figure 2B), respectively. As for ZFAS1, AUCs of platelets and plasma were $0.780(95 \% \mathrm{CI}=0.701-0.858, p<0.0001)$ and $0.806(95 \% \mathrm{CI}=0.731-0.881, p<0.0001)$ in $\mathrm{AD}$ (Figure $2 \mathrm{C})$, and $0.744(95 \% \mathrm{CI}=0.641-0.848, p<0.0001)$ and 0.770 $(95 \% \mathrm{CI}=0.663-0.878, p<0.0001)$ in SCC (Figure 2D). Of course, combination of two lncRNAs possessed a better ability for discrimination between NSCLC and controls, both in platelets and plasma, and the AUCs of the combined MAGI2$A S 3$ and $Z F A S 1$ were $0.908(95 \% \mathrm{CI}=0.853-0.963, p<0.0001)$ in platelets and $0.890(95 \% \mathrm{CI}=0.834-0.946, p<0.0001)$ in plasma of AD (Figure 2E).The AUCs of the combined MAGI2-AS3 and ZFAS1 were 0.919 (95\% CI $=0.848-0.990$, $p<0.0001)$ in platelets and $0.902(95 \% \mathrm{CI}=0.833-0.972$, $p<0.0001$ ) in plasma of SCC (Figure 2F). As for the AUCs of combinations, please see details in Table S2.

\section{EGFR intracellular mutations detection with platelets and plasma DNA}

Fourteen NSCLC patients with AD were diagnosed with $E G F R$ intracellular mutations with conventional tissue analysis. We extracted DNA of platelets and corresponding plasma from the 14 patients and detected $E G F R$ intracellular mutations. Three plasma samples (NO. 6, NO. 11, and NO. 12 listed in Table S3) showed positive result in accordance with the result of tissue while there was no positive result in platelets (Figure 3). As for negative result and basic information of 14 patients, please see summary in Table S3.

\section{Platelets and plasma from NSCLC patients contain mRNA EGFRvIII}

To determine whether circulating blood platelets and plasma isolated from NSCLC patients contain the RNA biomarker EGFRvIII, we compared platelets and plasma from $110 \mathrm{ADs}$ and 95 SCCs to 50 healthy people. Among 205 NSCLC patients, GAPDH was detected in all blood platelets and 


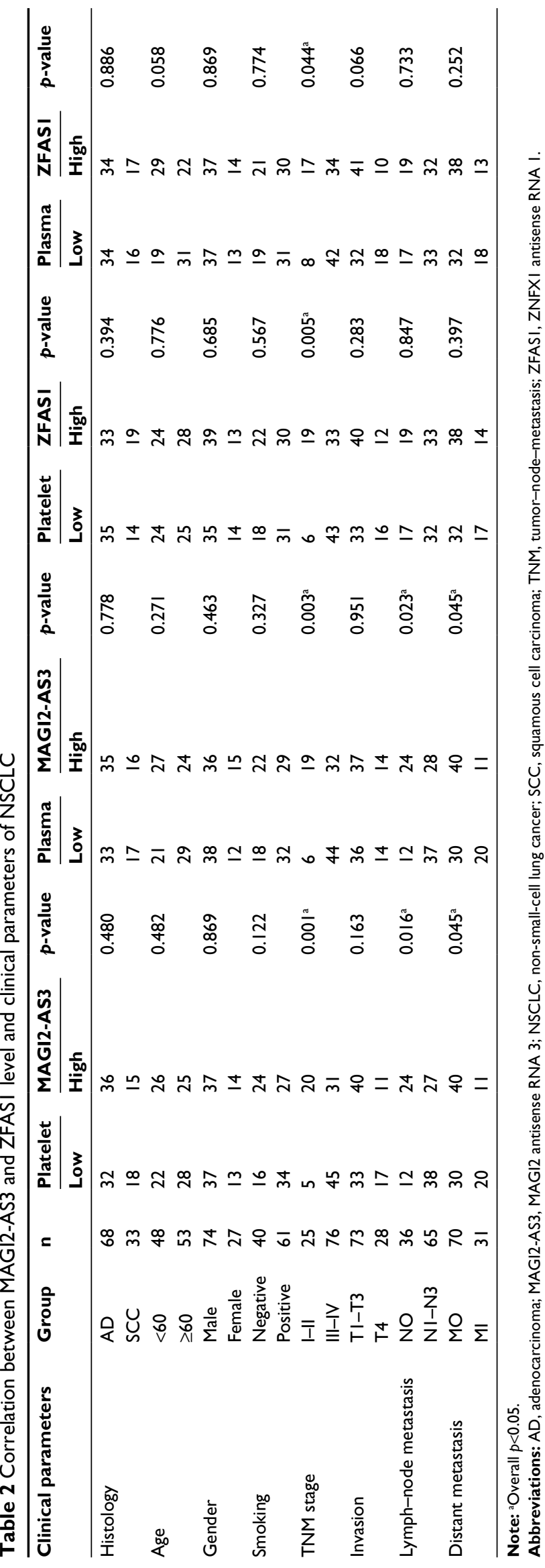

plasma samples, and EGFRvIII was detected in three (one in ADs and two in SCC) blood platelets and the corresponding plasma samples (Figure 4A, B). In addition, among 50 healthy people, none of the blood platelets and plasma samples detected EGFRVIII expression, but GAPDH was detected in all blood platelets and plasma samples (Figure 4C, D). Our data suggested that EGFRvIII can also be detected in blood platelets and there was no difference with detection in plasma. To verify whether EGFRvIII expression of platelets was contaminated by plasma, we performed the following analysis. First, we mixed the EGFRvIII-positive plasma and EGFRvIII-negative platelets, then separated platelets and detected EGFRvIII expression, and the result showed that there was not EGFRvIII expression in platelets (Figure S3A). Second, we mixed the EGFRvIII-positive platelets and EGFRvIII-negative plasma, then separated plasma and detected EGFRvIII expression, and the result showed that there was not EGFRvIII expression in plasma (Figure S3B). Taken together, these results suggested that the EGFRvIII expression we detected was specific to platelets.

\section{Discussion}

For decades, systematic empirical research on NSCLC has identified some prognostic factors and several biomarkers. ${ }^{27}$ However, the prognosis of NSCLC remains quite poor and the 5-year survival rate for NSCLC patients is low, because most patients are diagnosed at an advanced stage of NSCLC. Recently, the clinical application of liquid biopsy in NSCLC progressively proved a pivotal tool for screening and early detection of cancer, ${ }^{28}$ including TEPs, exosomes, circulating cell-free tumor DNA, and circulating tumor cells.

Here, we have examined the use of TEPs and plasma for a noninvasive assessment of IncRNAs and found that TEP is also a promising biosource similar to plasma. We investigated the clinical value of MAGI2-AS3, LOC100507632, FOXF1$A S 1$, LOC100499467, and ZFAS1. However, we could not detect LOC100507632, FOXF1-AS1, and LOC100499467 which might be due to the low abundance in platelets and plasma, suggesting that not all of the lncRNAs can be detected in platelets. As for MAGI2-AS3, NSCLC patient platelets and plasma were significantly downregulated in line with the results from GEO. For ZFAS1, previous study ${ }^{29}$ suggested that ZFAS1 is significantly downregulated in human breast cancer, where it serves as a tumor suppressor gene in tumorigenesis and progression, while another study ${ }^{30}$ showed that ZFAS1 is frequently amplified in hepatocellular carcinoma and functions as an oncogene. Thus, the roles of ZFAS1 in cancers are complex and might play a dual role depending 


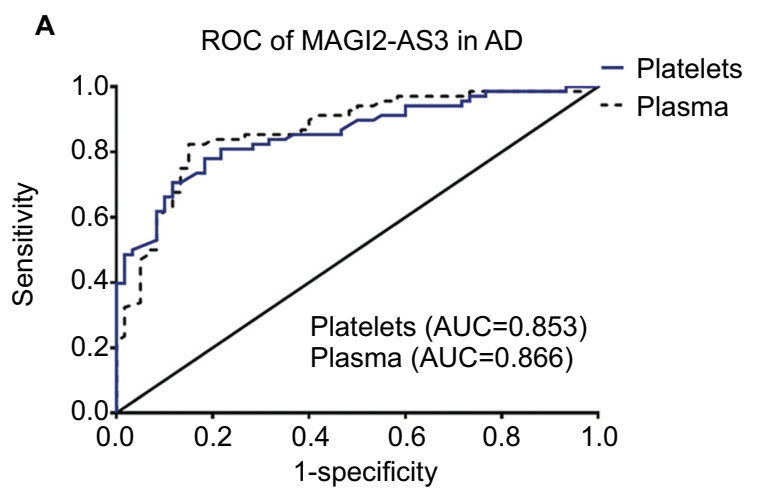

C

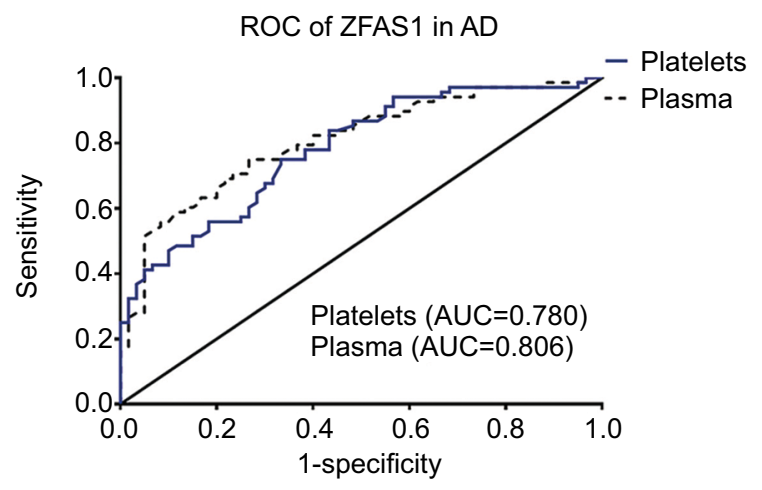

E

ROC of merged MAGI2-AS3 and AS1 in AD

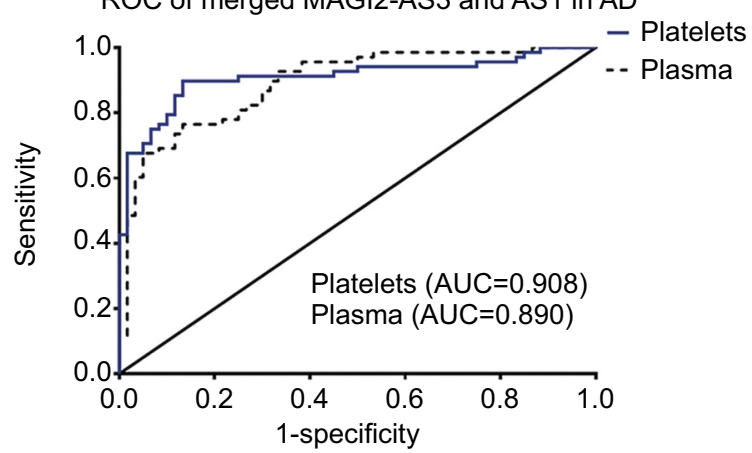

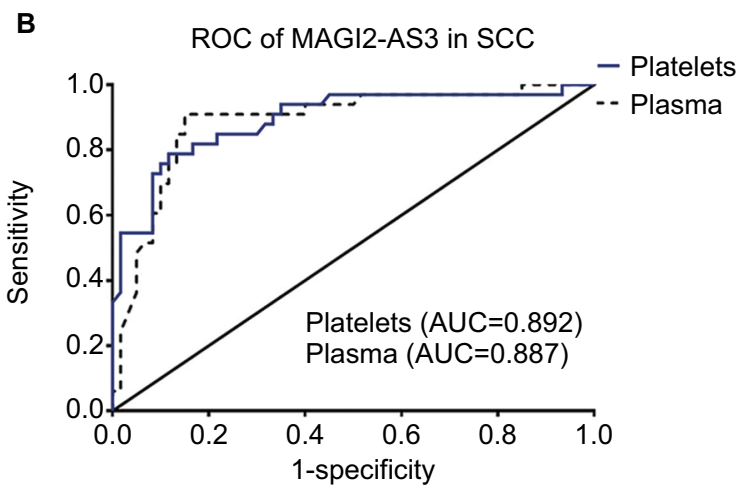

D

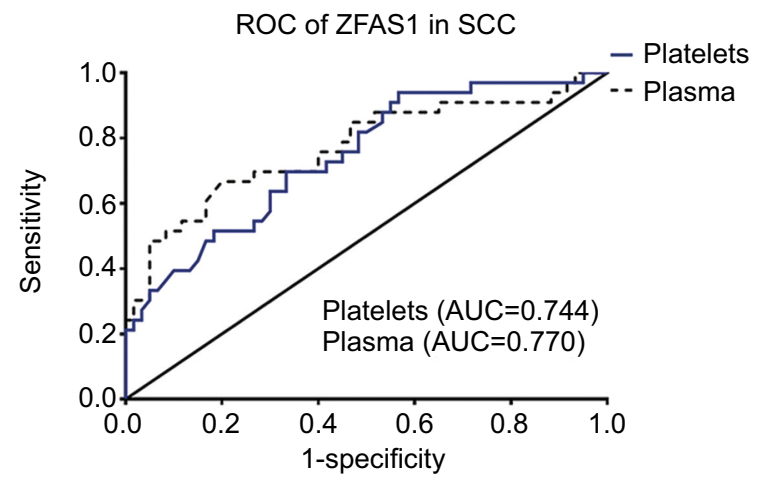

$\mathbf{F}$

ROC of merged MAGI2-AS3 and ZFAS1 in SCC

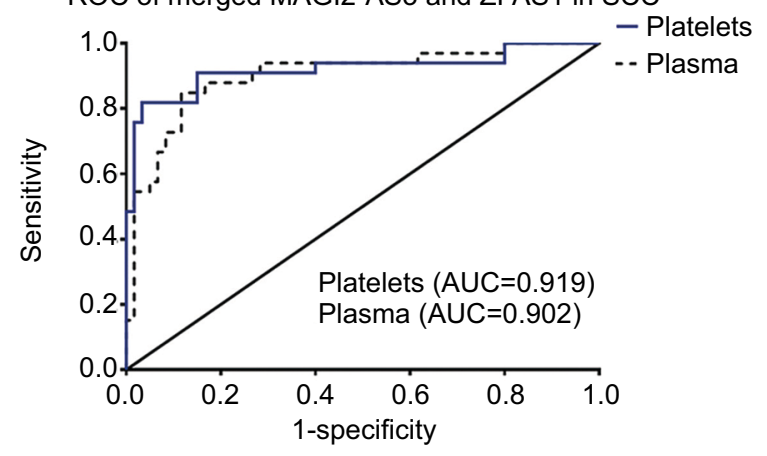

Figure 2 Diagnostic value of MAGI2-AS3 and ZFASI in adenocarcinoma and SCC.

Notes: (A) The ROC curve analysis for the diagnostic value of MAGI2-AS3 in platelets from adenocarcinoma $(\mathrm{AUC}=0.853,95 \% \mathrm{Cl}=0.789-0.918, p<0.000 \mathrm{I})$ and $(\mathrm{AUC}=0.866$, $95 \% \mathrm{Cl}=0.802-0.929, p<0.000 \mathrm{I}$ ) in plasma. (B) The ROC curve analysis for the diagnostic value of MAGl2-AS3 in platelets from SCC (AUC $=0.892,95 \% \mathrm{Cl}=0.819-0.965$, $p<0.0001$ ) and ( $A \cup C=0.887,95 \% \mathrm{Cl}=0.813-0.961, p<0.000 \mathrm{I}$ ) in plasma. (C) The ROC curve analysis for the diagnostic value of $Z F A S I$ in platelets from adenocarcinoma ( $A \cup C=0.780,95 \% \mathrm{Cl}=0.70 \mathrm{I}-0.858, p<0.000 \mathrm{I}$ ) and ( $\mathrm{AUC}=0.806,95 \% \mathrm{Cl}=0.73 \mathrm{I}-0.88 \mathrm{I}, p<0.000 \mathrm{I}$ ) in plasma. (D) The ROC curve analysis for the diagnostic value of $Z F A S I$ in platelets from SCC (AUC=0.744, 95\% Cl=0.64I-0.848, $p<0.000 \mathrm{I})$ and $(\mathrm{AUC}=0.770,95 \% \mathrm{Cl}=0.663-0.878, p<0.000 \mathrm{I})$ in plasma. (E) The ROC curve analysis for the diagnostic value of merged MAGI2-AS3 and ZFASI in platelets from adenocarcinoma ( $\mathrm{AUC}=0.908,95 \% \mathrm{Cl}=0.853-0.963, p<0.000 \mathrm{I}$ ) and $(\mathrm{AUC}=0.890,95 \% \mathrm{Cl}=0.834-0.946, p<0.000 \mathrm{I}$ ) in plasma. (F) The ROC curve analysis for the diagnostic value of merged MAGI2-AS3 and ZFASI in platelets from SCC (AUC $=0.919,95 \% \mathrm{Cl}=0.848-0.990, p<0.000 \mathrm{I})$ and (AUC $=0.902,95 \% \mathrm{Cl}=0.833-0.972$, $p<0.000 \mathrm{I}$ ) in plasma.

Abbreviations: AD, adenocarcinoma; AUC, area under the ROC curve; MAGI2-AS3, MAGI2 antisense RNA 3; ROC, receiver operating characteristic; SCC, squamous cell carcinoma; ZFASI, ZNFXI antisense RNA I.

on the tumor. It has previously been demonstrated that other small noncoding RNAs, such as microRNAs, can play a dual role as an oncogene or a tumor suppressor gene according to the cellular context. ${ }^{31}$ In addition, piRNAs can also play a dual role depending on the tumor. For example, piR-823 has been shown to act as a tumor suppressor in gastric cancer ${ }^{32}$ 
A
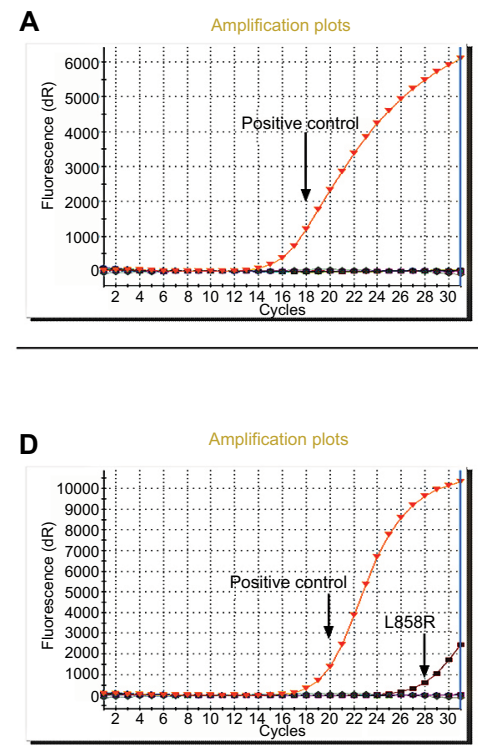

B

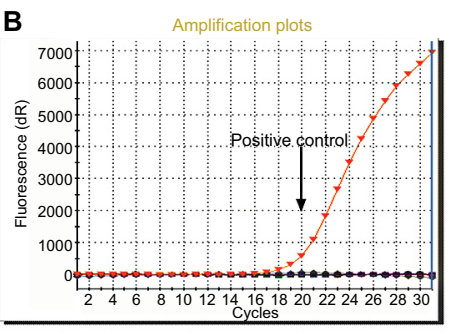

Platelets

E

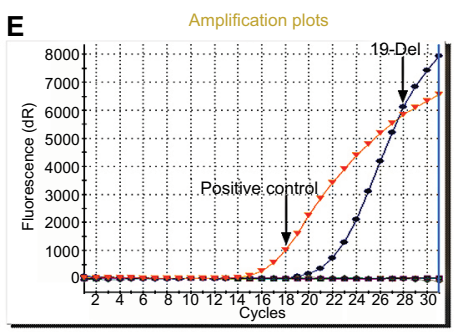

Plasma
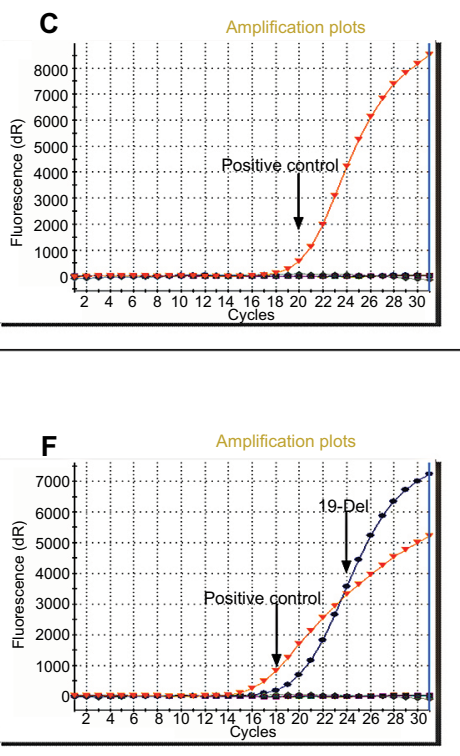

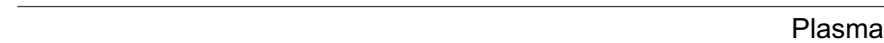

Figure 3 ARMS-PCR for EGFR intracellular mutations detection.

Notes: (A), (B), (C) EGFR intracellular mutation cannot be detected in NO.6, NO.II, and NO.I2 patient platelets. (D), (E), and (F) EGFR intracellular mutation can be detected in NO.6, NO.I I, and NO.I 2 patient plasma.

Abbreviations: ARMS, amplification-refractory mutation system; EGFR, epidermal growth factor receptor.
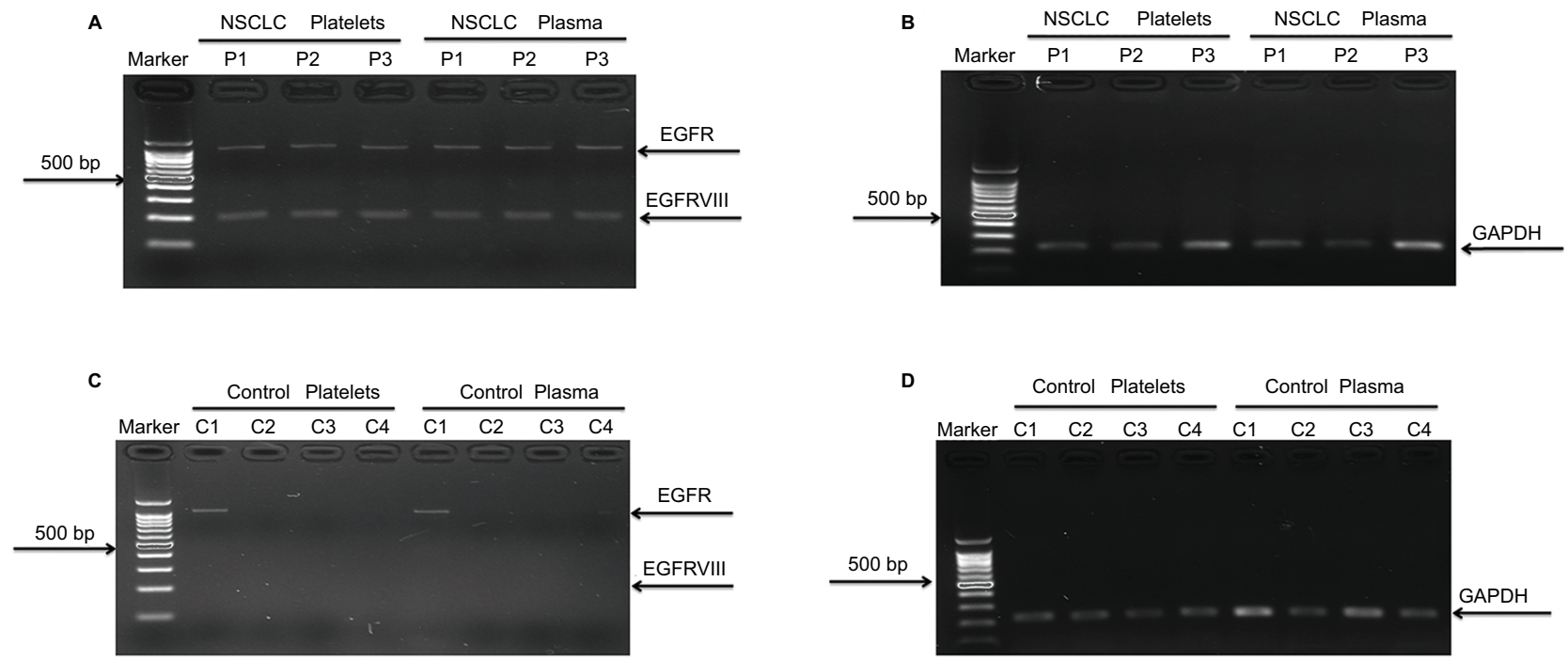

Figure 4 EGFRvIll expression in platelets and plasma derived from NSCLC patients.

Notes: (A) EGFRvIll expression in 3 NSCLC platelets and plasma. (B) GAPDH expression as positive control in patients' platelets and plasma, three controls' results are shown here as an example. (C) No EGFRvIll expression in 50 healthy controls, and four individuals' results are shown here as an example. (D) GAPDH expression as positive control in healthy individuals' platelets and plasma. Four individuals' results are shown here as an example.

Abbreviations: EGFR, epidermal growth factor receptor; NSCLC, non-small-cell lung cancer.

and as an oncogene in multiple myeloma. ${ }^{33}$ In our study, we found that ZFAS1 was significantly downregulated in platelets and plasma, which was in contrast to the high expression in NSCLC tissue samples as demonstrated in previous study, ${ }^{34}$ and this phenomenon might be explained by the expression of ZFAS1 detected in plasma and platelets which could have come from circulating cells rather than from tumor cells.
Meanwhile, we analyzed the diagnostic value of ZFAS1 and MAGI2-AS3 and found that molecular interrogation of platelet lncRNA ZFAS1 and MAGI2-AS3 can offer valuable diagnostics information for NSCLC patients analyzed, of both MAGI2-AS3 (AUC is 0.853 in AD and 0.892 in SCC) and ZFASI (AUC is 0.780 in AD and 0.744 in SCC). More importantly, combination of two lncRNAs possessed a better 
ability for discrimination between NSCLC and controls. We also analyzed the correlation between plasma lncRNA and platelet lncRNA, and we found that there was moderate correlation between both $M A G I 2-A S 3$ ( $r=0.738, p<0.0001)$ and ZFAS1 ( $r=0.751, p<0.0001)$. Furthermore, we found the correlation between the two lncRNAs' level and the clinicopathologic characteristics of the 101 NSCLC cancer patients and found that the expression level of ZFAS1 is negatively correlated to the TNM stage while MAGI2-AS3 expression significantly correlated with TNM stage, lymph-node metastasis, and distant metastasis. These findings suggested that the use of TEPs as a biosource to diagnose cancer may enable earlier diagnosis of cancer, improve convenience for NSCLC patients, and ultimately serve as supplement for clinical oncologic decision-making.

TEP is an emerging source for liquid biopsy, confrontation of platelets with tumor cells through transfer of tumorassociated biomolecules and results in the sequestration of such biomolecules, ${ }^{35,36}$ as shown in Figure 5. Numerous studies have emphasized the role of platelets in tumor biology, from a diagnostic standpoint of view, and TEPs carry important tumor molecular signature, with the potential to be used as a viable biosource for companion diagnostics and therapy selection.

In the present study, we extracted DNA of 14 platelets and plasma samples to detect EGFR intracellular mutations including 19-Del, L858R, T790M, 20-lns, G719X, S768I, and $L 861 Q$ by ARMS; our data suggested that only three plasma samples could detect EGFR mutation in accordance with tissue while there was no positive result in platelets, which also indicated there was no contamination of platelet DNA by plasma DNA, and the reasons that we analyzed to explain it are as follows: first, DNA in the blood of cancer patients is derived from cells that disintegrate by necrosis and apoptosis in expanding tumor tissue, ${ }^{37}$ and this contributed to the results of plasma that were in accordance with the tissue; second, platelets reserve megakaryocyte-derived cytoplasmic pre-mRNA, some of which are spliced into mRNA, ${ }^{38}$ but most of them are microRNAs accounting for $80 \% ;{ }^{39}$ third, platelets can take up tumor-derived membrane vesicles such as exosomes that contain tumor-associated biomarkers; ${ }^{16,35}$ however, tumor cells might tend to exploit circulating RNA as a means to "communicate" with their regional or distal environment than the DNA derived from

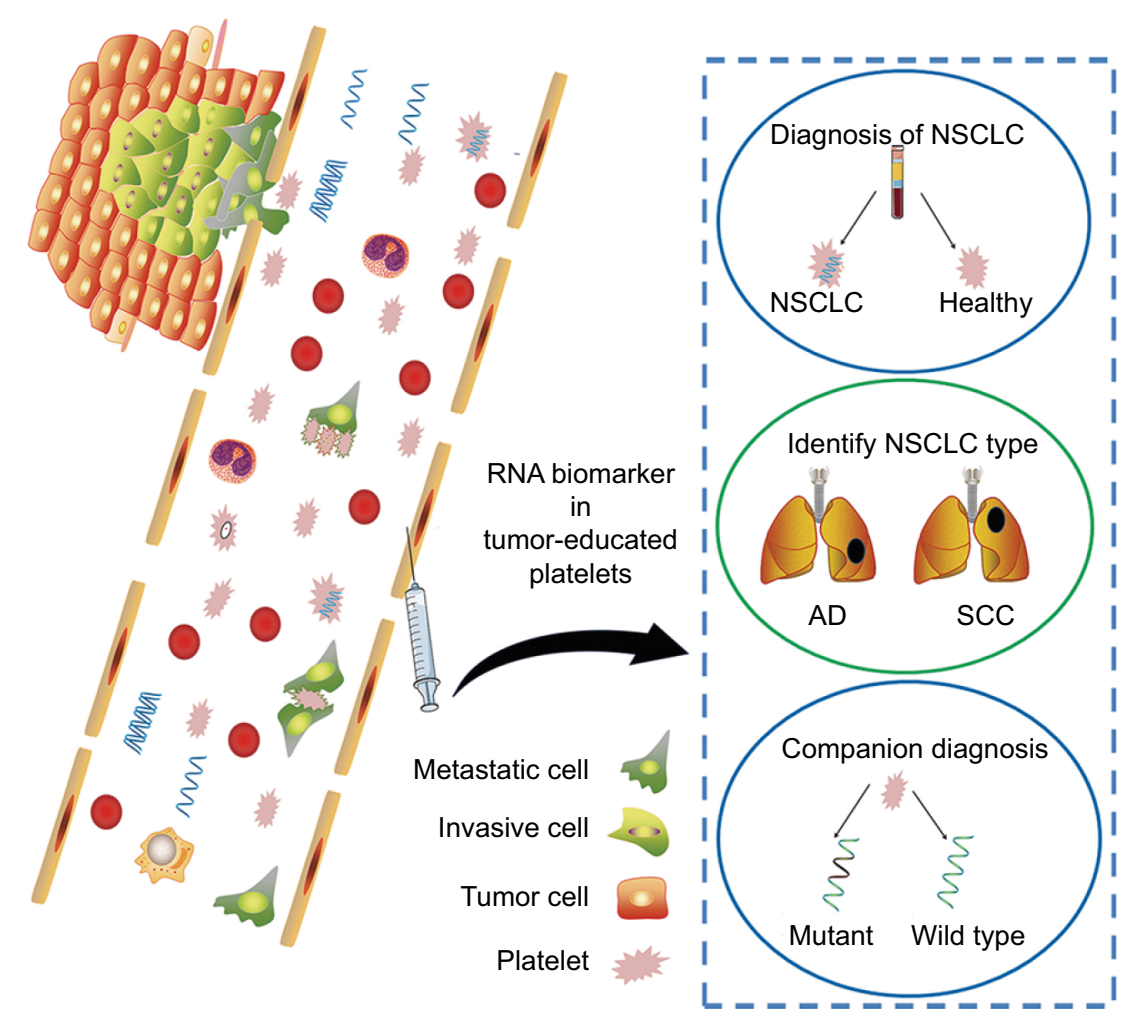

Figure 5 A schematic figure summarizing potential role of platelets in NSCLC.

Notes: Platelets communicate with tumor cells through uptaking of tumor-associated biomolecules and thus may serve as a potential diagnostic marker for NSCLC. Abbreviations: AD, adenocarcinoma; NSCLC, non-small-cell lung cancer; SCC, squamous cell carcinoma. 
necrosis or apoptosis. ${ }^{15,40}$ Finally, the detection sensitivity of the ADx-ARMS EGFR mutation detection kit we used might be low.

Then, we shifted the focus of research to RNA of platelets. A previous study ${ }^{16}$ has shown that blood platelets isolated from glioma patients contain the tumor-associated RNA biomarker EGFR $I I I$, and EGFRvIII has an in-frame deletion of the extracellular domain and is found in numerous types of human tumors. As EGFRvIII has been reported to be tumor specific and has oncogenic potential, it has been shown to be an extremely attractive target for anticancer therapy and is more sensitive to TK inhibition. ${ }^{41}$ Duan et al ${ }^{42}$ found EGFR$v I I I$ in eight of $114(7.0 \%)$ in tissues of NSCLC patients. Therefore, we detected the EGFR $I I I$ expression in TEPs and the corresponding plasma isolated from NSCLC; EGFRvIII was detected in three of 205 (1.5\%) patients, including $2.1 \%$ (2/95) SCC and $0.9 \%(1 / 110) \mathrm{AD}$, and there was no difference with EGFR $I I I$ detection between platelets and plasma. The data suggested that platelets are closely related to NSCLC and platelets contain tumor-derived RNA biomarker, which was consistent with the previous report. ${ }^{16}$

\section{Conclusion}

Our data suggested that TEP is a promising biosource to enable diagnosis of NSCLC, companion diagnostics of NSCLC, and improve convenience for NSCLC patients.

\section{Acknowledgments}

This work was supported by Applied Basic Research Program of Science and Technology Bureau Foundation of Wuhan (No. 2016060101010054) and National Natural Science Foundation of China (No. 81672114). This work was also funded by Science and Technology Innovation Fostering Foundation of Zhongnan Hospital of Wuhan University (cxpy20160025) and Wuhan City health and family planning medical talented youth development project.

\section{Disclosure}

The authors report no conflicts of interest in this work.

\section{References}

1. Pastorino U. Lung cancer screening. Br J Cancer. 2010;102(12): 1681-1686.

2. Seve P, Reiman T, Dumontet $\mathrm{C}$. The role of betaIII tubulin in predicting chemoresistance in non-small cell lung cancer. Lung Cancer. 2010;67(2):136-143.

3. Siegel R, Naishadham D, Jemal A. Cancer statistics, 2013. CA Cancer J Clin. 2013;63(1):11-30.

4. Jones PA, Baylin SB. The epigenomics of cancer. Cell. 2007;128(4): 683-692.
5. National Lung Screening Trial Research Team; Aberle DR, Adams AM, Berg CD, et al. Reduced lung-cancer mortality with low-dose computed tomographic screening. N Engl J Med. 2011;365(5):395-409.

6. Bruske-Hohlfeld I. Environmental and occupational risk factors for lung cancer. Methods Mol Biol. 2009;472:3-23.

7. Torre LA, Siegel RL, Ward EM, Jemal A. Global cancer incidence and mortality rates and trends-an update. Cancer Epidemiol Biomarkers Prev. 2016;25(1):16-27.

8. Crowley E, Di Nicolantonio F, Loupakis F, Bardelli A. Liquid biopsy: monitoring cancer-genetics in the blood. Nat Rev Clin Oncol. 2013;10(8):472-484.

9. Alix-Panabieres C, Schwarzenbach H, Pantel K. Circulating tumor cells and circulating tumor DNA. Annu Rev Med. 2012;63:199-215.

10. Haber DA, Velculescu VE. Blood-based analyses of cancer: circulating tumor cells and circulating tumor DNA. Cancer Discov. 2014;4(6):650-661.

11. Azmi AS, Bao B, Sarkar FH. Exosomes in cancer development, metastasis, and drug resistance: a comprehensive review. Cancer Metastasis Rev. 2013;32(3-4):623-642.

12. Pietras K, Ostman A. Hallmarks of cancer: interactions with the tumor stroma. Exp Cell Res. 2010;316(8):1324-1331.

13. Zhang Z, Ramnath N, Nagrath S. Current status of CTCs as liquid biopsy in lung cancer and future directions. Front Oncol. 2015;5:209.

14. Joosse SA, Pantel K. Tumor-educated platelets as liquid biopsy in cancer patients. Cancer Cell. 2015;28(5):552-554.

15. Nilsson RJ, Karachaliou N, Berenguer J, et al. Rearranged EML4-ALK fusion transcripts sequester in circulating blood platelets and enable blood-based crizotinib response monitoring in non-small-cell lung cancer. Oncotarget. 2016;7(1):1066-1075.

16. Nilsson RJ, Balaj L, Hulleman E, et al. Blood platelets contain tumorderived RNA biomarkers. Blood. 2011;118(13):3680-3683.

17. Yuana Y, Sturk A, Nieuwland R. Extracellular vesicles in physiological and pathological conditions. Blood Rev. 2013;27(1):31-39.

18. Spizzo R, Almeida MI, Colombatti A, Calin GA. Long non-coding RNAs and cancer: a new frontier of translational research? Oncogene. 2012;31(43):4577-4587.

19. Enfield KS, Pikor LA, Martinez VD, Lam WL. Mechanistic roles of noncoding RNAs in lung cancer biology and their clinical implications. Genet Res Int. 2012;2012:737416.

20. Ren S, Wang F, Shen J, et al. Long non-coding RNA metastasis associated in lung adenocarcinoma transcript 1 derived miniRNA as a novel plasma-based biomarker for diagnosing prostate cancer. Eur J Cancer. 2013;49(13):2949-2959.

21. Wu Y, Liu H, Shi X, Yao Y, Yang W, Song Y. The long non-coding RNA HNF1A-AS1 regulates proliferation and metastasis in lung adenocarcinoma. Oncotarget. 2015;6(11):9160-9172.

22. Gutschner T, Hammerle M, Eissmann M, et al. The noncoding RNA MALAT1 is a critical regulator of the metastasis phenotype of lung cancer cells. Cancer Res. 2013;73(3):1180-1189.

23. Shigematsu H, Lin L, Takahashi T, et al. Clinical and biological features associated with epidermal growth factor receptor gene mutations in lung cancers. J Natl Cancer Inst. 2005;97(5):339-346.

24. Maemondo M, Inoue A, Kobayashi K, et al; North-East Japan Study Group. Gefitinib or chemotherapy for non-small-cell lung cancer with mutated EGFR. $N$ Engl J Med. 2010;362(25):2380-2388.

25. Rosell R, Carcereny E, Gervais R, et al; Spanish Lung Cancer Group in collaboration with Groupe Français de Pneumo-Cancérologie and Associazione Italiana Oncologia Toracica. Erlotinib versus standard chemotherapy as first-line treatment for European patients with advanced EGFR mutation-positive non-small-cell lung cancer (EURTAC): a multicentre, open-label, randomised phase 3 trial. Lancet Oncol. 2012;13(3):239-246.

26. Yang J, Lin J, Liu T, et al. Analysis of IncRNA expression profiles in non-small cell lung cancers (NSCLC) and their clinical subtypes. Lung Cancer. 2014;85(2):110-115.

27. Wang LP, Niu H, Xia YF, et al. Prognostic significance of serum sMICA levels in non-small cell lung cancer. Eur Rev Med Pharmacol Sci. 2015;19(12):2226-2230. 
28. Perez-Callejo D, Romero A, Provencio M, Torrente M. Liquid biopsy based biomarkers in non-small cell lung cancer for diagnosis and treatment monitoring. Transl Lung Cancer Res. 2016;5(5):455-465.

29. Askarian-Amiri ME, Crawford J, French JD, et al. SNORD-host RNA $\mathrm{Zfas} 1$ is a regulator of mammary development and a potential marker for breast cancer. $R N A$. 2011;17(5):878-891.

30. Li T, Xie J, Shen C, et al. Amplification of long noncoding RNA ZFAS1 promotes metastasis in hepatocellular carcinoma. Cancer Res. 2015;75(15):3181-3191.

31. Schotte D, Pieters R, Den Boer ML. MicroRNAs in acute leukemia: from biological players to clinical contributors. Leukemia. 2012;26(1):1-12.

32. Cheng J, Deng H, Xiao B, et al. piR-823, a novel non-coding small RNA, demonstrates in vitro and in vivo tumor suppressive activity in human gastric cancer cells. Cancer Lett. 2012;315(1):12-17.

33. Yan H, Wu QL, Sun CY, et al. piRNA-823 contributes to tumorigenesis by regulating de novo DNA methylation and angiogenesis in multiple myeloma. Leukemia. 2015;29(1):196-206.

34. Tian FM, Meng FQ, Wang XB. Overexpression of long-noncoding RNA ZFAS1 decreases survival in human NSCLC patients. Eur Rev Med Pharmacol Sci. 2016;20(24):5126-5131.

35. Best MG, Sol N, Kooi I, et al. RNA-Seq of tumor-educated platelets enables blood-based pan-cancer, multiclass, and molecular pathway cancer diagnostics. Cancer Cell. 2015;28(5):666-676.
36. Kanikarla-Marie P, Lam M, Menter DG, Kopetz S. Platelets, circulating tumor cells, and the circulome. Cancer Metastasis Rev. 2017;36(2): 235-248.

37. Jahr S, Hentze H, Englisch S, et al. DNA fragments in the blood plasma of cancer patients: quantitations and evidence for their origin from apoptotic and necrotic cells. Cancer Res. 2001;61(4):1659-1665.

38. DenisMM,TolleyND,BuntingM, etal.Escapingthenuclearconfines: signaldependent pre-mRNA splicing in anucleate platelets. Cell. 2005;122(3) 379-391.

39. Ple H, Landry P, Benham A, Coarfa C, Gunaratne PH, Provost P. The repertoire and features of human platelet microRNAs. PLoS One. 2012;7(12):e50746.

40. Mittelbrunn M, Gutierrez-Vazquez C, Villarroya-Beltri C, et al. Unidirectional transfer of microRNA-loaded exosomes from $\mathrm{T}$ cells to antigen-presenting cells. Nat Commun. 2011;2:282.

41. Azuma M, Danenberg KD, Iqbal S, et al. Epidermal growth factor receptor and epidermal growth factor receptor variant III gene expression in metastatic colorectal cancer. Clin Colorectal Cancer. 2006;6(3):214-218.

42. Duan J, Wang Z, Bai H, et al. Epidermal growth factor receptor variant III mutation in Chinese patients with squamous cell cancer of the lung. Thorac Cancer. 2015;6(3):319-326.
Cancer Management and Research

\section{Publish your work in this journal}

Cancer Management and Research is an international, peer-reviewed open access journal focusing on cancer research and the optimal use of preventative and integrated treatment interventions to achieve improved outcomes, enhanced survival and quality of life for the cancer patient. The manuscript management system is completely online and includes

\section{Dovepress}

a very quick and fair peer-review system, which is all easy to use. Visit http://www.dovepress.com/testimonials.php to read real quotes from published authors. 